\title{
MR Imaging in Patients with Suspected Liver Metastases: Value of Liver-Specific Contrast Agent Gadoxetic Acid
}

Kyung Hee Lee, $M D^{1}$, Jeong Min Lee, $M D^{1}$, Ji Hoon Park, $M D^{2}$, Jung Hoon Kim, $M D^{1}$, Hee Sun Park, $M D^{3}$, Mi Hye Yu, MD ${ }^{1}$, Jeong-Hee Yoon, MD' ${ }^{1}$, Joon Koo Han, MD' ${ }^{1}$, Byung Ihn Choi, MD

${ }^{1}$ Division of Abdominal Imaging, Department of Radiology, Seoul National University College of Medicine, Seoul 110-744, Korea; ${ }^{2}$ Division of Abdominal Imaging, Department of Radiology, Seoul National University Bundang Hospital, Seongnam 463-767, Korea; ${ }^{3}$ Department of Radiology, Konkuk University School of Medicine, Seoul 143-701, Korea

Objective: To compare the diagnostic performance of gadoxetic acid-enhanced magnetic resonance (MR) imaging with that of triple-phase multidetector-row computed tomography (MDCT) in the detection of liver metastasis.

Materials and Methods: Our institutional review board approved this retrospective study and waived informed consent. The study population consisted of 51 patients with hepatic metastases and 62 patients with benign hepatic lesions, who underwent triple-phase MDCT and gadoxetic acid-enhanced MRI within one month. Two radiologists independently and randomly reviewed MDCT and MRI images regarding the presence and probability of liver metastasis. In order to determine additional value of hepatobiliary-phase (HBP), the dynamic-MRI set alone and combined dynamic-and-HBP set were evaluated, respectively. The standard of reference was a combination of pathology diagnosis and follow-up imaging. For each reader, diagnostic accuracy was compared using the jackknife alternative free-response receiver-operatingcharacteristic (JAFROC).

Results: For both readers, average JAFROC figure-of-merit (FOM) was significantly higher on the MR image sets than on the MDCT images: average FOM was 0.582 on the MDCT, 0.788 on the dynamic-MRI set and 0.847 on the combined HBP set, respectively $(p<0.0001)$. The differences were more prominent for small $(\leq 1 \mathrm{~cm})$ lesions: average FOM values were 0.433 on MDCT, 0.711 on the dynamic-MRI set and 0.828 on the combined HBP set, respectively $(p<0.0001)$. Sensitivity increased significantly with the addition of HBP in gadoxetic acid-enhanced MR imaging $(p<0.0001)$.

Conclusion: Gadoxetic acid-enhanced MRI shows a better performance than triple-phase MDCT for the detection of hepatic metastasis, especially for small $(\leq 1 \mathrm{~cm})$ lesions.

Index terms: Gadoxetic acid; Magnetic resonance imaging; Computed tomography; Hepatic metastasis

Received February 14, 2013; accepted after revision August 4, 2013.

Corresponding author: Jeong Min Lee, MD, Department of Radiology and Institute of Radiation Medicine, Seoul National University College of Medicine, 101 Daehak-ro, Jongno-gu, Seoul 110-744, Korea.

- Tel: (822) 2072-3154 • Fax: (822) 743-6385

- E-mail: jmsh@snu.ac.kr

This is an Open Access article distributed under the terms of the Creative Commons Attribution Non-Commercial License (http://creativecommons.org/licenses/by-nc/3.0) which permits unrestricted non-commercial use, distribution, and reproduction in any medium, provided the original work is properly cited.

\section{INTRODUCTION}

In patients with diagnosed malignant disease, detection of liver metastasis is essential for optimal treatment planning at the time of diagnosis and during follow-up. As metastatic involvement of the liver in extrahepatic malignant disease may significantly change the therapeutic approach, it is important to rule out such involvement with a high confidence (1). Early identification of liver metastasis provides the opportunity for resection, which, at least in cases of colorectal carcinoma, has been shown 
to prolong patient survival (2). Imaging-guided interstitial therapies, including cryoablation, laser photo-coagulation, radiofrequency and microwave ablation and percutaneous ethanol injection, are currently evolving and may assume a curative or adjunctive role to other treatments. The success of such therapies depends on accurate imaging of liver neoplasms (3). Until now, both multidetector-row computed tomography (MDCT) and magnetic resonance (MR) imaging have been regarded as reliable imaging modalities for the diagnostic work-up of the liver. However, detection and characterization of small hepatic metastases continue to present diagnostic challenges. Differentiating small metastases and other incidental benign lesions, such as small cysts, hemangiomas, focal nodular hyperplasia and adenoma, is not always easy.

Gadoxetic acid (Primovist, Bayer Healthcare, Berlin, Germany) is a liver-specific MR contrast agent that has both dynamic and hepatocyte-specific properties (4-7). Gadoxetic acid (Gd-EOB-DTPA) contains an additional lipophilic chemical group that causes its uptake into hepatocytes followed by subsequent biliary excretion of approximately $50 \%$ in patients with normal renal and hepatic function (8). Once taken up by hepatocytes, gadoxetic acid enhances the hepatic parenchyma, whereas lesions with few or no hepatocytes such as cysts, metastases and most hepatocellular carcinomas remain unenhanced $(1,9,10)$. Using these properties of gadoxetic acid and the excellent spatial resolution of the three-dimensional, gradient-echo (3D GRE) sequence, gadoxetic acid-enhanced MRI may facilitate both the accurate detection and characterization of hepatocellular lesions, including hepatic metastases $(1,11-15)$. The use of gadoxetic acid in liver imaging has recently become popular with the promising results seen in recent studies evaluating focal liver lesions, especially the small lesions $(12,16)$. Nevertheless, the most clinically relevant problem when using gadoxetic acid for metastasis workup may not only be the ability to detect small lesions and but also to characterize them correctly, due to the difficulty in differentiating small hemangioma or small, benign, non-hepatocellular lesions from small metastases (17-21).

Therefore, the purpose of our study was to compare the diagnostic performance of gadoxetic acid-enhanced MR imaging with triple-phase MDCT for the detection of liver metastasis and to determine whether the addition of hepatobiliary phase (HBP) imaging to dynamic imaging can be helpful for the evaluation of liver metastases.

\section{MATERIALS AND METHODS}

\section{Patient Population}

This study was approved by the Institutional Review Board of Seoul National University Hospital and written informed consent was waived for all patients for the retrospective study. Between May 2008 and February 2009, 343 patients underwent gadoxetic-enhanced MRI for suspected liver metastasis. Among these patients, 199 were excluded for the following reasons: 1) there was no triple-phase, contrast-enhanced liver CT performed within one month before or after gadoxetic acid-enhanced MRI; or 2) there was insufficient proof of lesion etiology by either histopathology or surveillance. Of the remaining 144 patients, 80 had suspected liver metastasis seen on CT or MR imaging, while in 64 patients benign hepatic lesions were detected on both CT and MR imaging. Among the 80 patients with suspected liver metastasis, 29 were excluded from the study for the following reasons: they were undergoing or had undergone chemotherapy $(n=10)$; radiofrequency ablation was performed before pathologic confirmation $(n=9)$; there were more than 10 lesions in a single patient $(n=8)$; and CT or MR images were unavailable $(n=2)$. Meanwhile, among the 64 patients with suspected benign hepatic lesions, two were excluded from this study because liver metastases were newly observed at other locations on the follow-up images. Ultimately, 113 patients (patients with liver metastasis, $\mathrm{n}=51$; patients without liver metastasis, $n=62$ ) were included in the final study group. Our study population consisted of 72 males and 41 females $(M: F=1.8: 1)$ with age range of $30-86$ years (mean age and SD, $62.7 \pm 10.4$ years). Flow chart for the enrollment of the study population is presented in Figure 1.

\section{Standard of Reference}

The study coordinators with twenty, five and four years of clinical experience in abdominal radiology, respectively, decided in consensus regarding the presence or absence of hepatic metastasis, based on contrast-enhanced CT, US, gadoxetic acid-enhanced MR, follow-up US, CT, MR imaging and pathologic reports regarding the surgical or biopsy specimens. The proof of malignancy was based on either histopathology or imaging surveillance. During this review, 137 liver metastases were identified in 51 patients on the basis of: 1 ) surgery ( $n=63$ in 24 patients); 2) needle biopsy ( $n=14$ in 11 patients); or 3 ) tumor 


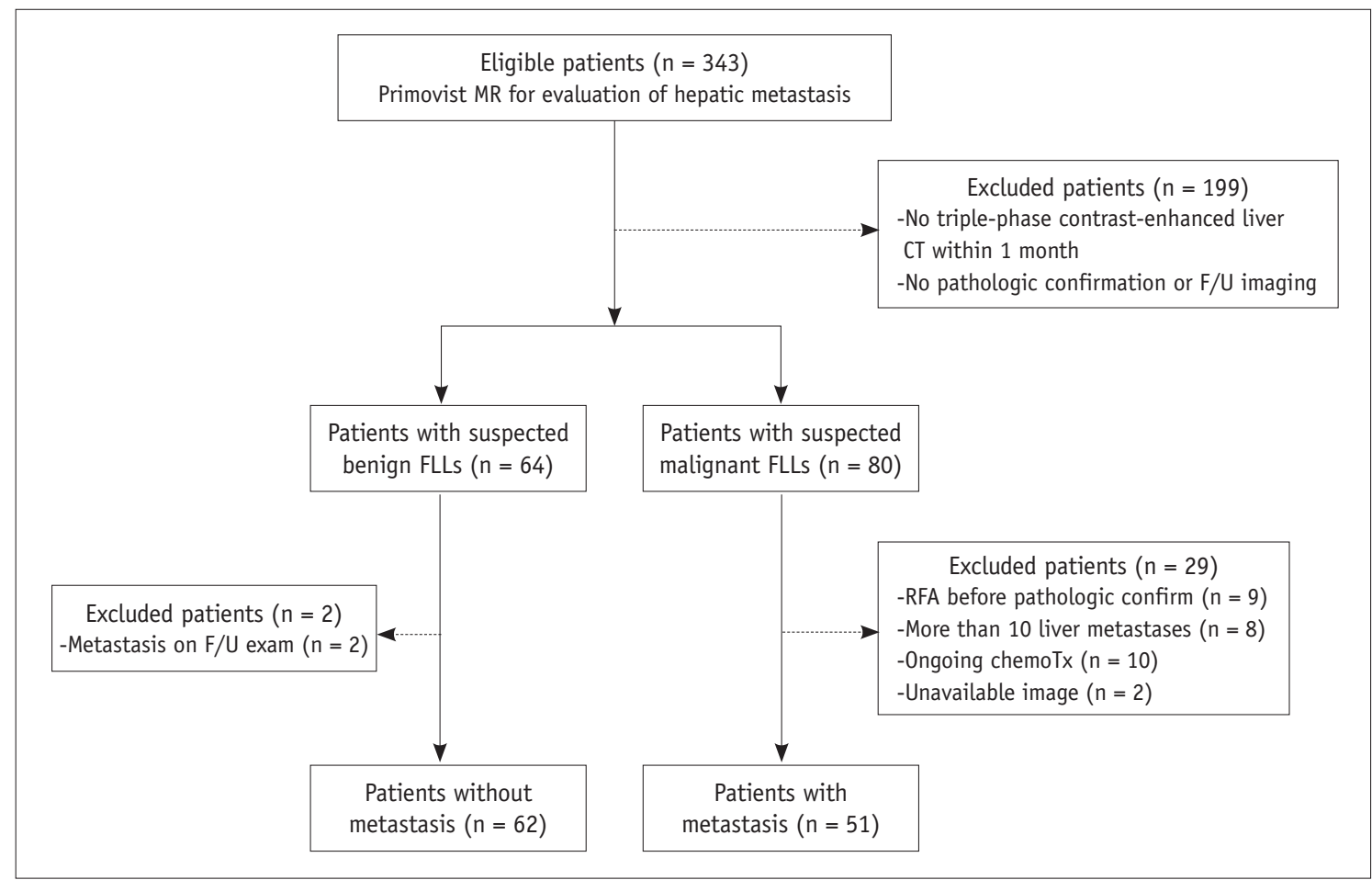

Fig. 1. Flow chart of inclusion criteria. Patients with and without liver metastasis were enrolled in evaluation of detectability of liver metastasis. RFA = radiofrequency ablation, $F L L=$ focal liver lesion, $F / U=$ follow-up

growth observed on follow-up, cross-sectional imaging ( $\mathrm{n}=60$ in 20 patients). Considering the possibility of concealed metastatic lesions, entire liver was screened with intraoperative ultrasonography during the surgery in our study population. Furthermore, all patients who underwent surgery or biopsy were followed-up with contrast-enhanced CT scan for minimum 6 months, and there was no additional recurrence. Without histopathology, liver metastasis was confirmed when: 1 ) the lesion showed the typical imaging findings of metastasis $(1,22-24)$ on at least two imaging modalities; and 2) interval growth in the longest axial diameter of at least $20 \%$, as seen on follow-up imaging. The mean follow-up interval was $8.3 \pm 5.0$ months (range: 4-24 months). For benign lesions, exclusion of interval growth seen on follow-up imaging was a prerequisite. Sixtytwo patients who had no hepatic metastasis were confirmed absence of hepatic metastases on follow-up imaging studies, performed at least six months following the initial MR imaging (25).

\section{Imaging Protocol}

\section{CT Protocol}

Contrast-enhanced, triple-phase, helical CT exams were performed in our institution on a 4-MDCT scanner (Mx
8000, Philips Medical Systems, Cleveland, OH, USA) in 18 patients, on a 16-MDCT scanner (LightSpeed Ultra, GE Healthcare, Milwaukee, WI, USA and Sensation 16, Siemens, Forchheim, Germany) in 28 patients, on a 64-MDCT scanner (Brilliance 64, Philips Healthcare, Cleveland, OH, USA) in 45 patients and on a dual-source CT (Somatom Definition, Siemens, Forchheim, Germany) scanning in the singleenergy mode in 13 patients. Nine patients were scanned at an outside hospital on MDCT scanners. At our institution, the scanning parameters were $120 \mathrm{kVp}, 189-200 \mathrm{mAs}, 5-\mathrm{mm}$ slice thickness with an increment (overlap) of $2.5 \mathrm{~mm}$, table speed of $18.75-26.75 \mathrm{~mm} /$ rotation (pitch, 0.828 1.07) and a single-breath-hold helical acquisition of 5-8 seconds, depending on the liver size. The images were obtained in the craniocaudal direction. A total of $1.5 \mathrm{~mL} / \mathrm{kg}$ of $370 \mathrm{mgI} / \mathrm{mL}$ of the nonionic contrast material, iopromide (Ultravist 370; Schering, Berlin, Germany), was injected into an antecubital vein at a rate of 2.0-4.0 mL/sec using a power injector (Stellent Dual; MEDRAD Inc., Warrendale, PA, USA). The arterial phase imaging was performed 19 seconds after attaining $100 \mathrm{HU}$ attenuation of the descending aorta, as measured using a bolus tracking technique. A 33-second delay after the arterial phase was obtained for the portal venous phase (PVP) acquisition (approximately a 70-second delay after contrast injection). The reconstructed section 
thickness was equal to or less than $5 \mathrm{~mm}$.

\section{MRI Protocol}

MRI was performed on a 3T General Electric scanner (Signa Excite HD, GE Healthcare, Milwaukee, WI, USA) and using an 8-element, torso phased array coil $(26,27)$. All MR images were obtained in the axial plane. A rectangular field of view of 320 to $350 \mathrm{~mm}$ was adjusted for each patient's body size and was held constant for all sequences. Baseline MR images were composed of a respiratory-triggered, T2weighted, rapid-acquisition, relaxation-enhanced (RARE; fast spin-echo) sequence, a half-Fourier acquisition, RARE sequence (single-shot fast spin-echo) and a breath-hold, T1-weighted, GRE sequence. Dynamic imaging including arterial phase, PVP and delayed phase, as well as HBP imaging, was performed using the same fat-suppressed three-dimensional GRE sequence (liver acquisition with volume acceleration [LAVA]; GE Medical Systems, Milwaukee, WI, USA) after administration of gadoxetic acid. The parameters of each pulse sequence are summarized in Table 1. After unenhanced imaging, all patients received a rapid bolus of 10-mL gadoxetic acid (Primovist, Bayer Schering Pharma AG, Berlin, Germany) at a rate of $1.5 \mathrm{~mL} / \mathrm{s}$, followed immediately by a $20-\mathrm{mL}$ saline flush through an antecubital venous catheter, using a power injector. Scanning delay times were determined using real-time MRI fluoroscopic monitoring following contrast administration (Carebous [Siemens] or SmartPrep [GE Medical Systems]). Hepatic AP images were obtained 7 seconds after contrast arrived at the distal thoracic aorta. Subsequent PVP, delayed-phase and HBP images were obtained at 60 seconds, 3 minutes and 20 minutes, respectively, after beginning the contrastmedium injection. Acquisition of 3D-LAVA data for each phase was finished during a single breath-hold at the end of expiration (time range, 18-22 seconds; mean time, 21 seconds) $(26,27)$.

\section{Image Interpretation and Analysis}

Image interpretation was conducted to evaluate diagnostic performance of CT and MR imaging for depicting liver metastasis. All interpretations were performed independently by two radiologists (with 13 and 10 years, respectively, of clinical experience in abdominal radiology). The radiologists were blinded to the patients' clinical histories and final diagnoses. All interpretations were performed using a commercially available viewer system or picture archiving and communication system (Maroview,

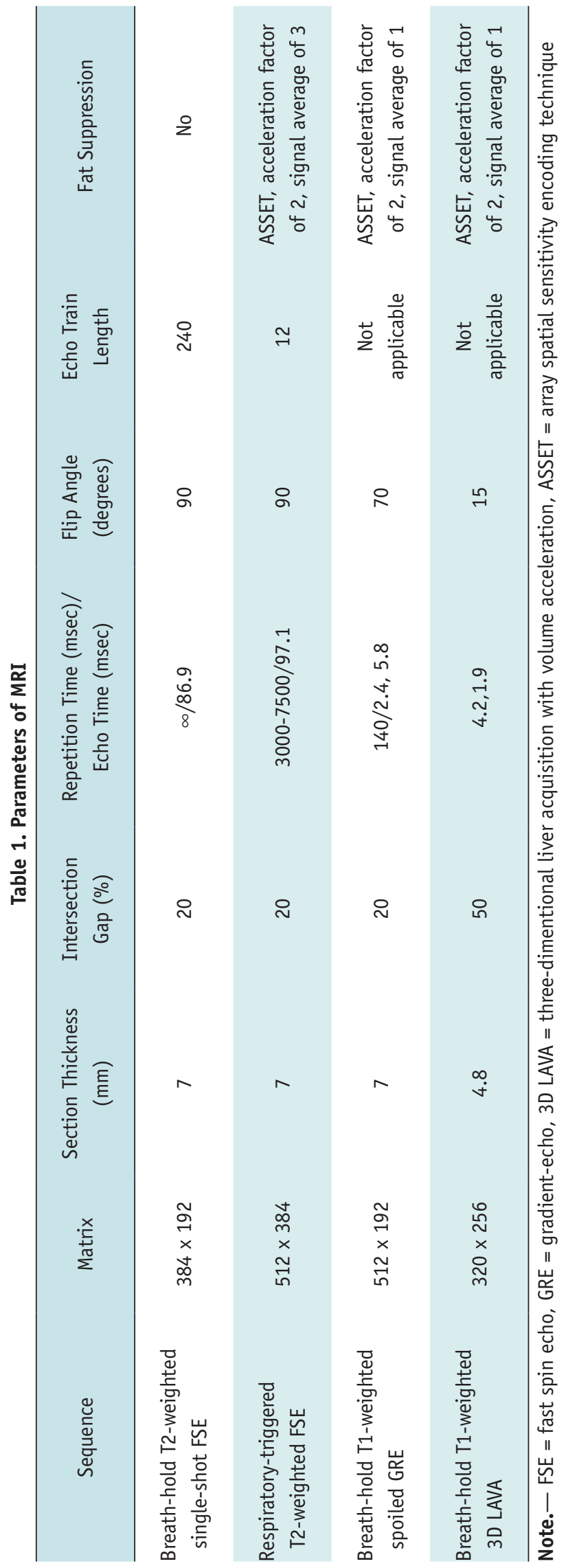


Marotech, Seoul, Korea).

Image interpretation was performed during two sessions, i.e., triple-phase MDCT and gadoxetic acid-enhanced MRI. More than a six-week time interval was set between the $\mathrm{CT}$ and MR reading sessions in order to reduce recall bias. All patient-related details were removed from the images, which were then provided in a random order to enable a patient-by-patient analysis. In the CT interpretation session, the two readers independently recorded the presence and probability of liver metastasis based on integration of the precontrast, arterial-, and portal-phase images. During the MRI interpretation, dynamic MRI set (precontrast T1-, T2weighted images and dynamic images) was first evaluated. Following this evaluation, HBP images were provided for the same patient in order to re-evaluate liver metastasis on a combined HBP set consisting of precontrast T1-, T2weighted images, dynamic images and 20-minute delay HBP images. The two readers assessed probability of liver metastasis for each patient, on both the dynamic MRI and combined HBP set.

For focal liver lesions, each reader recorded the size and location of the lesions and graded the probability of malignancy on a five-point confidence scale: 1 , definitely benign; 2 , probably benign; 3 , indeterminate; 4 , probably metastasis; and 5, definitely metastasis. The readers were informed that the lesions assigned to grades 4 or 5 were regarded as positive for metastasis. If two or more liver metastases were considered to be present in the liver of a single patient, the size, location and confidence score were reported on a review-sheet so that the study coordinators could identify the lesions pointed out by the readers in a lesion-by-lesion analysis. If any false-positive or falsenegative results were observed in a reader's interpretation, the study coordinators also assessed the reason for the misinterpretation by reviewing the CT and MR images.

\section{Statistical Analysis}

In the statistical analysis, the data of each reader were analyzed separately in order to avoid within-reader clustering. Jackknife FROC, especially developed to analyze the observer free-response tasks (28-30), was used to compare the diagnostic performance of $\mathrm{CT}$ and MR imaging for depicting liver metastasis. Jackknife FROC software (JAFROC, version 4.1; http://www.devchakraborty.com) $(29,31)$ was used to compute a figure-of-merit (FOM). The FOM is defined as the probability that lesions, including unmarked lesions, are rated higher than non-lesion marks on control liver CT or MRI (28); or in other words, that lesions are given a higher confidence rating for the presence of malignancy than normal findings. Both normal images with no marks and unmarked lesions are assigned a zero rating. To calculate the sensitivity, specificity, positive predictive value (PPV) and negative predictive value (NPV) for each modality, lesions assigned to grades 4 or 5 were regarded as positive for the presence of liver metastasis. The McNemar test was used to compare the sensitivity and specificity of $\mathrm{CT}$ and MR imaging, whereas the Fisher exact test was used to compare PPV and NPV. Subgroup analysis for lesion-by-lesion analysis was also performed on lesions equal to or less than $1 \mathrm{~cm}$ in diameter. In the patient-bypatient analysis, the readers' assessment was assumed as positive when at least one lesion was detected in the liver. $\kappa$ values were calculated to assess inter-reader variability for detecting liver metastasis on $\mathrm{CT}$ and MR, respectively, both on a per-patient and per-lesion basis. The inter-reader agreement for the presence of metastasis was estimated on the patient-basis, and that in the confidence scale was calculated on the lesion-basis. A $\kappa$ value of $0.81-1.00$ was interpreted as excellent; $0.61-0.80$, substantial agreement; 0.41-0.60, moderate agreement; 0.21-0.40, fair agreement; and $0.00-0.20$, poor agreement. All statistical analyses were performed using a commercially available software (SPSS, version 17.0; SPSS Inc., Chicago, IL, USA). For all studies, a difference with a $p$ value less than 0.05 was considered significant.

\section{RESULTS}

\section{Demographic Characteristics}

The demographic and clinical characteristics of the benign and metastasis groups are shown in Table 2. No significant differences in patient age (Mann-Whitney $U$ test, $p>0.05$ ), patient sex (Fisher exact test, $p>0.05$ ) and the interval between CT and MR examinations (Mann-Whitney $\mathrm{U}$ test, $p$ $>0.05$ ) were seen between those patients with and without liver metastasis.

The mean size of the liver metastasis was $11.17 \pm 10.47$ $\mathrm{mm}$ (range, 3-70 mm). In 51 patients with liver metastasis, the primary cancer sites and types included colorectal adenocarcinoma $(n=38)$, stomach $(n=4)$, breast $(n=$ $2)$, pancreas $(n=2)$, Ampulla of Vater $(n=2), G B(n=1)$, ovary $(n=1)$ and rectal carcinoid tumor $(n=1)$.

In 62 patients without liver metastasis, 100 benign hepatic lesions were identified including cysts $(n=53)$, 
Table 2. Demographic and Clinical Characteristics of Patients with and without Liver Metastases

\begin{tabular}{lccc}
\hline & Patients without Liver Metastasis & Patients with Liver Metastasis & $P$-Value \\
\hline No. of patients & $62(55 \%)$ & $51(45 \%)$ & \\
Mean age $^{*}$ & $63.1 \pm 11.1$ & $62.3 \pm 9.6$ & $0.70^{\dagger}$ \\
Male-to-female ratio $^{\dagger}$ & $38: 24$ & $34: 17$ & $0.55^{\ddagger}$ \\
CT-MR interval (days) $^{*}$ & $11.8 \pm 11.6$ & $13.5 \pm 10.2$ & $0.41^{\dagger}$ \\
\hline
\end{tabular}

Note. - * Data are mean values \pm standard deviations, ${ }^{\dagger}$ Calculated using Mann-Whitney $\mathrm{U}$ test, ${ }^{\ddagger}$ Calculated using Fisher exact test.

Table 3. Diagnostic Performance for Detection of Liver Metastases: Patient-by-Patient Analysis

\begin{tabular}{|c|c|c|c|c|c|c|}
\hline \multirow{2}{*}{ Statistic and Reviewer } & \multicolumn{3}{|c|}{ Patient-by-Patient Analysis } & \multicolumn{3}{|c|}{$P$-Value } \\
\hline & $\mathrm{CT}^{\mathrm{a}}$ & Dynamic MRI Set ${ }^{b}$ & Combined HBP Set $^{c}$ & $a$ vs. $b$ & a vs. c & b vs. c \\
\hline \multicolumn{7}{|l|}{ Sensitivity (\%)* } \\
\hline Reviewer 1 & $80.4(41 / 51)$ & $90.2(46 / 51)$ & $92.2(47 / 51)$ & 0.18 & 0.11 & 1 \\
\hline Reviewer 2 & $76.5(39 / 51)$ & $90.2(46 / 51)$ & $96.1(49 / 51)$ & 0.07 & 0.01 & 0.25 \\
\hline \multicolumn{7}{|l|}{ Specificity (\%)* } \\
\hline Reviewer 1 & $71.0(44 / 62)$ & $90.3(56 / 62)$ & $85.5(53 / 62)$ & 0.004 & 0.049 & 0.38 \\
\hline Reviewer 2 & $72.8(47 / 62)$ & $79.0(49 / 62)$ & $80.7(50 / 62)$ & 0.75 & 0.58 & 1 \\
\hline \multicolumn{7}{|l|}{$\operatorname{PPV}(\%)^{\dagger}$} \\
\hline Reviewer 1 & $69.5(41 / 59)$ & $88.5(46 / 52)$ & $83.9(47 / 56)$ & 0.02 & 0.08 & 0.58 \\
\hline Reviewer 2 & $72.2(39 / 54)$ & $78.0(46 / 59)$ & $80.3(49 / 61)$ & 0.52 & 0.38 & 0.82 \\
\hline \multicolumn{7}{|l|}{$\operatorname{NPV}(\%)^{\dagger}$} \\
\hline Reviewer 1 & $81.5(44 / 54)$ & $91.8(56 / 61)$ & $93.0(53 / 57)$ & 0.16 & 0.09 & 1 \\
\hline Reviewer 2 & $79.7(47 / 59)$ & $90.7(49 / 54)$ & $96.2(50 / 52)$ & 0.12 & 0.01 & 0.44 \\
\hline
\end{tabular}

Note. - Data in parentheses are numbers used to calculate percentages. ${ }^{*}$ Calculated using McNemar test, ${ }^{\dagger}$ Calculated using Fisher exact test. $\mathrm{HBP}=$ hepatobiliary-phase, $\mathrm{PPV}=$ positive predictive value, $\mathrm{NPV}=$ negative predictive value

unclassified benign nodules (i.e., eosinophilic infiltration) $(n=22)$, hemangiomas $(n=15)$, pseudolesions due to partial volume averaging or other artifacts $(n=7)$, focal fat deposition $(n=1)$, calcification $(n=1)$ and air bubble $(n=1)$.

\section{Detection of Liver Metastasis}

In the patient-by-patient analysis, all four of the diagnostic performance parameters (sensitivity, specificity, PPV and NPV), were higher on the MR imaging than on CT (Table 3). The combined HBP set revealed a higher sensitivity and NPV than the dynamic MRI set for both readers.

In the lesion-by-lesion analysis, both readers saw higher JAFROC FOM values with the gadoxetic acid-enhanced MR images, and the average JAFROC FOM was 0.582 on CT images, 0.788 on the dynamic MRI set and 0.847 on the combined HBP set $(p<0.0001)$ (Table 4, Fig. 2). The combined HBP set had a significantly better sensitivity (92.0\% for Reader 1 and $81.8 \%$ for Reader 2), than the dynamic MRI set $(79.6 \%$ for Reader 1 and $70.8 \%$ for Reader $2, p<0.0001)$ and the triple-phase MDCT images $(58.4 \%$ for Reader 1 and $45.3 \%$ for Reader $2, p<0.0001$ ). With the gadoxetic acid-enhanced HBP images, seventeen additional metastatic nodules (12.4\%) by Reader 1 and fifteen nodules
(11.0\%) by Reader 2 were seen compared to the number seen on the dynamic MRI set. All of these nodules were of a small size $(\leq 10 \mathrm{~mm})$. The NPVs were also highest with the combined HBP set by both Reader $1(p<0.0001)$ and Reader $2(p<0.0001)$ among the three image sets.

Among the 237 metastatic and benign hepatic lesions, $153(64.6 \%)$ were equal to or less $1 \mathrm{~cm}$. Differences in JAFROC FOM values and sensitivities were more prominent for lesions equal to or smaller than $10 \mathrm{~mm}$ in diameter (Table 5): the average JAFROC FOM and sensitivity were 0.433 and $26.6 \%$ on CT images; 0.711 and $60.8 \%$ on the dynamic MRI set; and 0.828 and $81.0 \%$ on the combined HBP set, respectively $(p<0.0001)$.

\section{False-Positive and False-Negative Findings}

The causes of false-negative and false-positive findings were assumed based on the retrospective review of the CT and MR images and the pathology results and in consensus by the two radiologists. We found that the majority of false-negative nodules detected on CT and MRI were of a small tumor size $\leq 10 \mathrm{~mm}$, as seen by both readers. $0 \mathrm{n}$ the CT examination, $89.5 \%(51 / 57)$ of the false-negative nodules undetected by Reader 1 and $86.7 \%$ (65/75) of the false-negative nodules undetected by Reader 2 were of a 
Table 4. Diagnostic Performance for Detection of Liver Metastases: Lesion-by-Lesion Analysis

\begin{tabular}{|c|c|c|c|c|c|c|}
\hline \multirow{2}{*}{ Statistic and Reader } & \multicolumn{3}{|c|}{ Lesion-by-Lesion Analysis: All Lesions } & \multicolumn{3}{|c|}{$P$-Value } \\
\hline & $\mathrm{CT}^{\mathrm{a}}$ & Dynamic MRI Set $^{b}$ & Combined HBP Set ${ }^{c}$ & $a$ vs. b & a vs. c & b vs. c \\
\hline \multicolumn{7}{|l|}{ JAFROC FOM* } \\
\hline Reader 1 & $0.615(0.508,0.723)$ & $0.842(0.777,0.906)$ & $0.902(0.847,0.957)$ & $<0.0001$ & $<0.0001$ & 0.05 \\
\hline Reader 2 & $0.548(0.461,0.635)$ & $0.733(0.643,0.822)$ & $0.792(0.729,0.867)$ & $<0.0001$ & $<0.0001$ & 0.04 \\
\hline \multicolumn{7}{|l|}{ Sensitivity $(\%)^{\dagger}$} \\
\hline Reader 1 & $58.4(80 / 137)$ & $79.6(109 / 137)$ & $92.0(126 / 137)$ & $<0.0001$ & $<0.0001$ & $<0.0001$ \\
\hline Reader 2 & $45.3(62 / 137)$ & $70.8(97 / 137)$ & $81.8(112 / 137)$ & $<0.0001$ & $<0.0001$ & $<0.0001$ \\
\hline \multicolumn{7}{|l|}{ Specificity $(\%)^{\dagger}$} \\
\hline Reader 1 & $72.0(72 / 100)$ & $92.0(92 / 100)$ & $81.0(81 / 100)$ & 0.001 & 0.16 & 0.003 \\
\hline Reader 2 & $83.0(83 / 100)$ & $81.0(81 / 100)$ & $80.0(80 / 100)$ & 0.82 & 0.66 & 1 \\
\hline \multicolumn{7}{|l|}{ PPV $(\%)^{\ddagger}$} \\
\hline Reader 1 & $74.1(80 / 108)$ & $93.2(109 / 117)$ & $86.9(126 / 145)$ & $<0.0001$ & 0.01 & 0.11 \\
\hline Reader 2 & $78.5(62 / 79)$ & $86.6(97 / 116)$ & $84.9(112 / 132)$ & 0.45 & 0.13 & 0.48 \\
\hline \multicolumn{7}{|l|}{ NPV $(\%)^{\ddagger}$} \\
\hline Reader 1 & $55.8(72 / 129)$ & $76.7(92 / 120)$ & $88.0(81 / 92)$ & 0.001 & $<0.0001$ & 0.048 \\
\hline Reader 2 & $52.3(83 / 158)$ & $66.9(81 / 121)$ & $76.2(80 / 105)$ & 0.02 & $<0.0001$ & 0.14 \\
\hline
\end{tabular}

Note. - Unless otherwise specified, data in parentheses are numbers used to calculate percentages. *Numbers in parentheses are $95 \%$ confidence interval, ${ }^{\dagger}$ Calculated using McNemar test, ${ }^{\ddagger}$ Calculated using Fisher exact test. JAFROC $=$ jackknife alternative free-response receiver-operating-characteristic, FOM = figure-of-merit, HBP = hepatobiliary-phase, PPV = positive predictive value, NPV = negative predictive value

small tumor diameter $(\leq 10 \mathrm{~mm})$. On the MR images, all of the false-negative results interpreted by the two readers were due to a small tumor size $\leq 10 \mathrm{~mm}$, except for four metastases. Of these four, two metastases $(13 \mathrm{~mm}, 20 \mathrm{~mm}$ in diameter) were not detected by either reader due to their sub-capsular location. For the remaining two nodules, the readers were able to identify them as two metastatic nodules but considered them as benign nodules. One nodule $(\mathrm{S} 6,12 \mathrm{~mm}$ ) was considered to be probably benign (score $2)$; and the other nodule $(S 7,16 \mathrm{~mm}$ ) was regarded as an indeterminate (score 3 ) nodule by both readers.

The mean diameter of the false-positive lesions was $12.0 \pm 10.3 \mathrm{~mm}$. There were 28,17 and 8 false positive nodules seen by Reader 1 on the CT, dynamic MRI set and combined HBP set, respectively. There were 19, 19 and 20 seen on the CT, dynamic MRI and combined HBP set by Reader 2. On the CT images, a total of 33 hepatic nodules were misinterpreted as metastasis: inflammatory nodules ( $\mathrm{n}$ $=14)$, hemangiomas $(n=8)$, cysts $(n=7)$, pseudolesions due to partial volume or artifacts $(n=3)$ and focal fat deposition $(n=1)$. On the dynamic MRI set, a total of 22 hepatic nodules were misclassified as metastatic nodules: inflammatory nodules $(n=10)$, hemangiomas $(n=7)$, cyst $(n=1)$, psuedolesions due to partial volume or artifacts ( $n$ $=3$ ) and an air bubble $(n=1)$. On the combined HBP set, a total of 27 hepatic nodules were misclassified as metastatic nodules: inflammatory nodules $(n=16)$, hemangiomas ( $n$
$=6)$, cyst $(n=1)$, psuedolesions due to partial volume averaging or other artifacts $(n=3)$ and an air bubble $(n=1)$.

\section{Inter-Reader Variability}

The weighted $\kappa$ value for the lesion-by-lesion analysis of liver metastasis was 0.65 (substantial agreement) for CT imaging and 0.56-0.57 (moderate agreement) for MR imaging for Readers 1 and 2. The weighted $\kappa$ value for the patient-by-patient analysis of liver metastasis was uniformly substantial ( $\kappa$ value, $0.71-0.78$ ) for both CT and MR imaging.

\section{DISCUSSION}

According to our study, the diagnostic performance of gadoxetic acid-enhanced dynamic and HBP MR imaging for detecting hepatic metastasis was superior to that of triplephase MDCT. Higher sensitivity and NPV were achieved with gadoxetic acid-enhanced MR imaging compared with those obtained with triple-phase MDCT imaging. Our results are, therefore, consistent with those of other published reports $(8,25)$, which demonstrated the higher diagnostic accuracy of gadoxetic acid-enhanced MRI than that of MDCT for distinguishing liver metastases from colorectal cancer. The major strength of our study over previous reports is that we included all consecutive liver metastasis patients, not only those limited to colorectal cancer. We also 


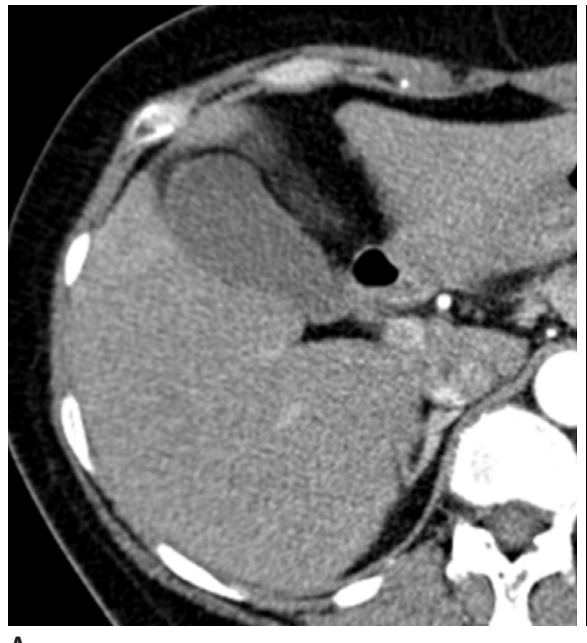

A

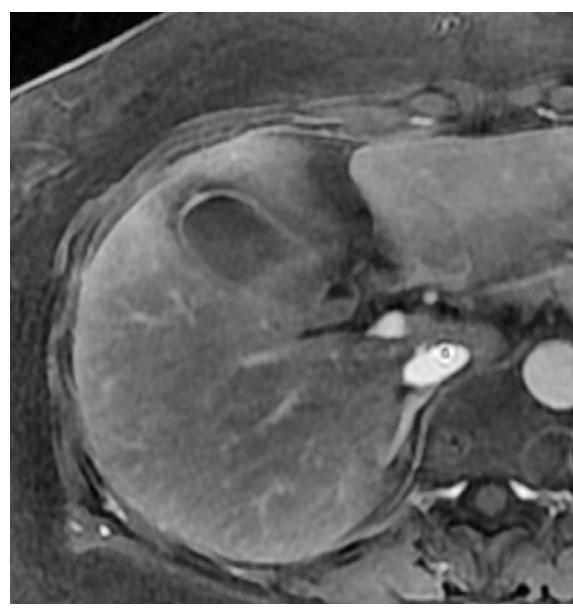

D
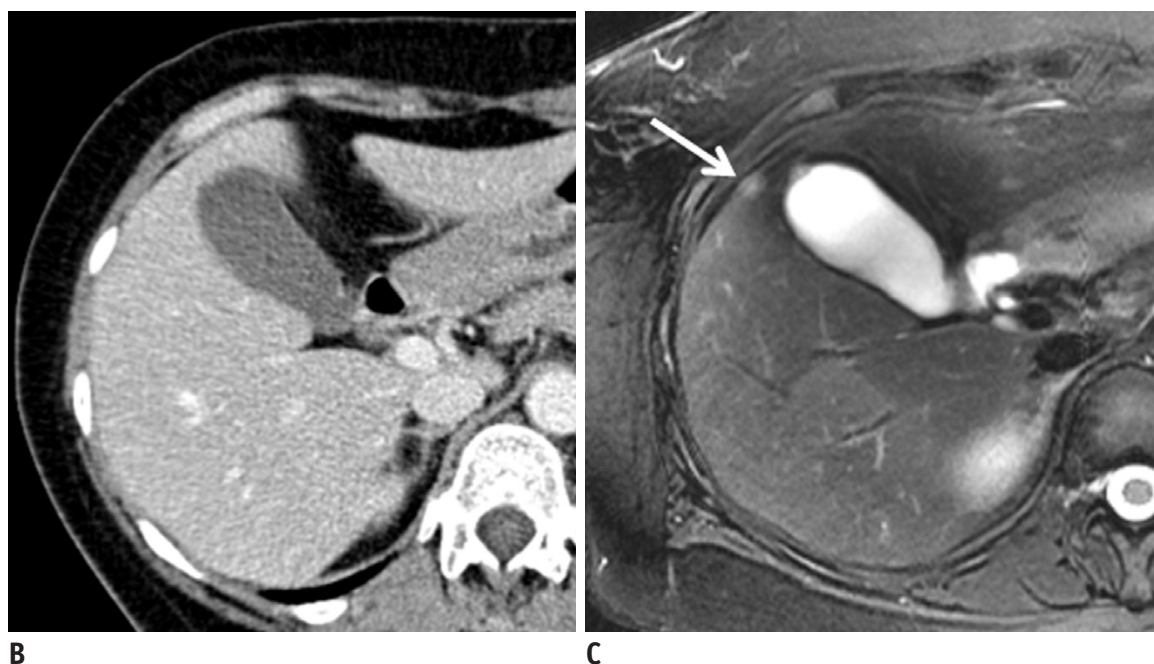

C

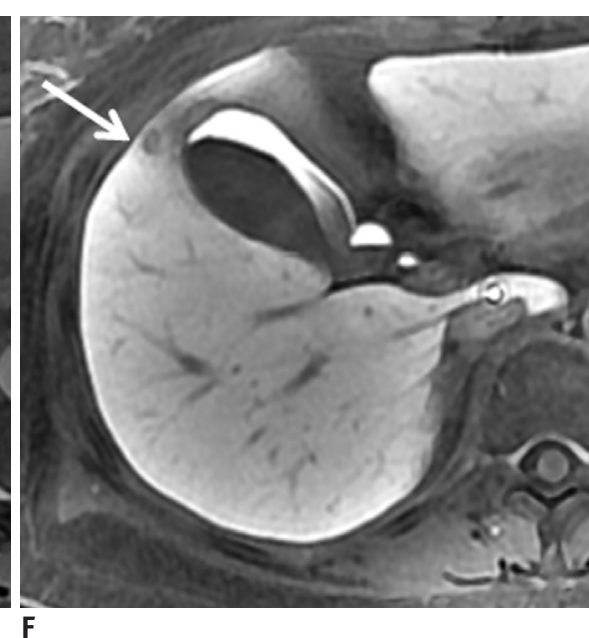

Fig. 2. Surgically proven liver metastasis from ascending colon cancer in 53-year-old woman.

A, B. Contrast-enhanced CT scans on arterial (A) and portal phase (B) show no definite liver metastasis. C. Respiratory-triggered, T2-weighted FSE image reveals one metastatic lesion in gallbladder bed (arrow). D, E. Arterial (D) and equilibrium (E) phase images after administration of gadoxetic-acid show lesion as faintly hypo-intense nodule (arrow). F. Gadoxetic-acid HBP MR image shows low-signal-intensity nodule in segment IV of liver (arrow). FSE = fast spin echo, HBP = hepatobiliary-phase

evaluated the added value of the HBP images for detection of liver metastasis. While our CT examinations revealed a limitation on localizing and characterizing small, lowattenuated hepatic nodules (such as small cysts or small, slowly enhancing hemangiomas), the combination of T2- or heavily T2-weighted images and dynamic and HBP images of the gadoxetic acid-enhanced MR examination resulted in a much more accurate lesion detection and characterization for both of our readers $(1,32)$.

In our study, the combined image analysis using the gadoxetic acid-enhanced dynamic images and HBP images allowed for a better diagnostic performance and offered a higher sensitivity for detecting hepatic metastases, when compared with image interpretation solely based on precontrast and dynamic MR imaging. The gadoxetic acid- enhanced HBP images were especially useful for detecting small hepatic metastases $1 \mathrm{~cm}$ in diameter or less; and with the HBP MR images, approximately $10 \%$ of hepatic metastases were additionally detected by both readers. Our study results were in good agreement with a recent study which showed better results for MR for detecting colorectal liver metastases than those for MDCT in patients who had the fatty liver after undergoing neoadjuvant chemotherapy (33). The high lesion-to-liver contrast of small metastases, as seen on HBP images of gadoxetic acid-enhanced MR imaging using a high-resolution 3D GRE technique, may have allowed for such successful results $(4,25,34)$. The high lesion-to-liver contrast of liver metastases seen on HBP images were derived from a strong, homogeneous enhancement of the liver parenchyma through the 
Table 5. Diagnostic Performance for Detection of Liver Metastases Equal to or Less than $1 \mathrm{~cm}$ in Diameter

\begin{tabular}{|c|c|c|c|c|c|c|}
\hline \multirow{2}{*}{ Statistic and Reader } & \multicolumn{3}{|c|}{ Lesion-by-Lesion Analysis: Lesions $\leq 10 \mathrm{~mm}$} & \multicolumn{3}{|c|}{$P$-Value } \\
\hline & $\mathrm{CT}^{\mathrm{a}}$ & Dynamic MRI Set ${ }^{b}$ & Combined HBP Set $^{\mathrm{C}}$ & a vs. b & a vs. $c$ & b vs. c \\
\hline \multicolumn{7}{|l|}{ JAFROC FOM* } \\
\hline Reader 1 & $0.461(0.333,0.589)$ & $0.757(0.669,0.846)$ & $0.878(0.800,0.954)$ & $<0.0001$ & $<0.0001$ & 0.01 \\
\hline Reader 2 & $0.404(0.287,0.521)$ & $0.664(0.518,0.810)$ & $0.777(0.663,0.892)$ & $<0.0001$ & $<0.0001$ & 0.007 \\
\hline \multicolumn{7}{|l|}{ Sensitivity $(\%)^{\dagger}$} \\
\hline Reader 1 & $35.4(28 / 79)$ & $68.4(54 / 79)$ & $89.9(71 / 79)$ & $<0.0001$ & $<0.0001$ & $<0.0001$ \\
\hline Reader 2 & $17.7(14 / 79)$ & $53.2(42 / 79)$ & $72.2(57 / 79)$ & $<0.0001$ & $<0.0001$ & $<0.0001$ \\
\hline \multicolumn{7}{|l|}{ Specificity $(\%)^{\dagger}$} \\
\hline Reader 1 & $75.7(56 / 74)$ & $91.9(68 / 74)$ & $75.7(56 / 74)$ & 0.02 & 1 & 0.002 \\
\hline Reader 2 & $90.5(67 / 74)$ & $82.4(61 / 74)$ & $79.7(59 / 74)$ & 0.18 & 0.06 & 0.5 \\
\hline \multicolumn{7}{|l|}{ PPV $(\%)^{\ddagger}$} \\
\hline Reader 1 & $60.9(28 / 46)$ & $90.0(54 / 60)$ & $79.8(71 / 89)$ & 0.001 & 0.02 & 0.12 \\
\hline Reader 2 & $66.7(14 / 21)$ & $76.4(42 / 55)$ & $79.2(57 / 72)$ & 0.40 & 0.25 & 0.83 \\
\hline \multicolumn{7}{|l|}{ NPV $(\%)^{\ddagger}$} \\
\hline Reader 1 & $52.3(56 / 107)$ & $73.1(68 / 93)$ & $87.5(56 / 64)$ & 0.003 & $<0.0001$ & 0.045 \\
\hline Reader 2 & $50.8(67 / 132)$ & $62.2(61 / 98)$ & $72.8(59 / 81)$ & 0.11 & 0.002 & 0.15 \\
\hline
\end{tabular}

Note. - Unless otherwise specified, data in parentheses are numbers used to calculate percentages. *Numbers in parentheses are $95 \%$ confidence interval, ${ }^{\dagger}$ Calculated using McNemar test, ${ }^{\ddagger}$ Calculated using Fisher exact test. JAFROC $=$ jackknife alternative free-response receiver-operating-characteristic, FOM = figure-of-merit, HBP $=$ hepatobiliary-phase, $\mathrm{PPV}=$ positive predictive value, NPV $=$ negative predictive value

hepatocyte-related uptakes seen during the HBP as well as the low degree of enhancement of those metastases without hepatocytes (35). Although the liver parenchyma also showed strong enhancement during the portal phase, the metastatic nodules also showed some degree of enhancement during the same phase, and, therefore, the contrast between the metastases and the liver parenchyma was less on portal phase images than on HBP images.

On the other hand, although the false positive rate of gadoxetic acid-enhanced MRI increased slightly with the addition of HBP, the readers still had difficulty differentiating inflammatory nodules or atypical hemangiomas from hepatic metastases. In our study, with the majority of hemangiomas showing a characteristic enhancement pattern (i.e., early peripheral nodular enhancement with centripetal progression) following the bolus injection of contrast material, the correct diagnosis was easily made by the two reviewers. However, several of the small, high-flow hepatic hemangiomas mimicked metastasis by showing hypointensity on delayed dynamic phase imaging, the so-called "pseudo washout sign", on gadoxetic acid-enhanced MRI. Such did not then allow for a confident diagnosis of hepatic hemangioma (17). This pseudo washout sign of hemangiomas should not be considered a true contrast washout, such as occurs in HCC or hypervascular metastasis. Rather it was thought to be caused by contrast uptake in the surrounding normal liver parenchyma. A close check for a high-signal intensity on heavily T2-weighted images or on high quality, diffusionweighted imaging (DWI) may, therefore, be helpful in differentiating small hemangiomas from metastases (21).

In our study, the sensitivity for detecting hepatic metastasis on CT was slightly lower than the recently reported sensitivity of dynamic CT for detecting hepatic metastasis. Most studies have reported a sensitivity between $60 \%$ and $80 \%(32,36-40)$. However, in our study, both of the readers who performed the image analysis were abdominal radiologists with more than 10 years of clinical experience in abdominal imaging. Therefore, we believe that the low CT sensitivity for hepatic metastasis may be associated with the high proportion (64.6\%) of small, hepatic nodules (diameter of $10 \mathrm{~mm}$ or less) seen in our study as well as the inherently low sensitivity of CT for detecting such tiny liver lesions.

Our study has several limitations. First, as our patient evaluation was retrospective, there was an unavoidable selection bias. Second, histopathology results were not obtained for all metastasis cases. However, as mentioned above, the three study coordinators carefully established the standard of reference based on the characteristic imaging findings of metastasis as well as the interval growth of those lesions as seen on follow-up examination. Third, we included triple-phase MDCT images obtained using various types of CT equipment. Despite this heterogeneity, 
we attempted to select the acceptable CT images for evaluation of possible liver metastasis, that is, those with adequate contrast enhancement and reconstructed section thickness equal to or less than $5 \mathrm{~mm}$. Fourth, although our study included patients with multiple liver metastases, perlesion sensitivity, specificity, PPV and NPV were compared using the McNemar test and Fisher's exact test. As we did not use the appropriate statistical method for correcting a possible statistical error resulting from the clustering effect, the results of this study may have a potential bias from the clustering effect. Finally, we did not include the DWI in evaluation of the diagnostic performance of MR examination in this study. Based on some recent studies $(18,41)$, which demonstrated that DWI was most valuable for identifying small metastases, we may anticipate a more precise localization and characterization of small hepatic metastases when using a combined reading of DWI and gadoxetic acid-enhanced MRI in clinical practice.

In conclusion, our study showed that gadoxetic acidenhanced MRI can provide a better diagnostic performance and higher sensitivity than triple-phase MDCT for the detection of liver metastases. Gadoxetic acid-enhanced MR images including HBP revealed a higher sensitivity for the detection of liver metastasis, especially for small lesions equal to or smaller than $10 \mathrm{~mm}$ in diameter.

\section{REFERENCES}

1. Zech CJ, Herrmann KA, Reiser MF, Schoenberg SO. MR imaging in patients with suspected liver metastases: value of liverspecific contrast agent Gd-EOB-DTPA. Magn Reson Med Sci 2007;6:43-52

2. Baker ME, Pelley R. Hepatic metastases: basic principles and implications for radiologists. Radiology 1995;197:329-337

3. Sica GT, Ji H, Ros PR. CT and MR imaging of hepatic metastases. AJR Am J Roentgenol 2000;174:691-698

4. Clemént 0 , Mühler $A$, Vexler VS, Kuwatsuru R, Berthezène $Y$, Rosenau W, et al. Comparison of Gd-EOB-DTPA and Gd-DTPA for contrast-enhanced MR imaging of liver tumors. J Magn Reson Imaging 1993;3:71-77

5. Halavaara J, Breuer J, Ayuso C, Balzer T, Bellin MF, Blomqvist $L$, et al. Liver tumor characterization: comparison between liver-specific gadoxetic acid disodium-enhanced MRI and biphasic CT--a multicenter trial. J Comput Assist Tomogr 2006;30:345-354

6. Huppertz A, Haraida S, Kraus A, Zech CJ, Scheidler J, Breuer $J$, et al. Enhancement of focal liver lesions at gadoxetic acid-enhanced MR imaging: correlation with histopathologic findings and spiral CT--initial observations. Radiology $2005 ; 234: 468-478$
7. Vogl TJ, Kümmel S, Hammerstingl R, Schellenbeck M, Schumacher G, Balzer $T$, et al. Liver tumors: comparison of MR imaging with Gd-EOB-DTPA and Gd-DTPA. Radiology 1996;200:59-67

8. Muhi A, Ichikawa T, Motosugi U, Sou H, Nakajima H, Sano K, et al. Diagnosis of colorectal hepatic metastases: comparison of contrast-enhanced CT, contrast-enhanced US, superparamagnetic iron oxide-enhanced MRI, and gadoxetic acid-enhanced MRI. J Magn Reson Imaging 2011;34:326-335

9. Hamm B, Staks T, Mühler A, Bollow M, Taupitz M, Frenzel $T$, et al. Phase I clinical evaluation of Gd-EOB-DTPA as a hepatobiliary MR contrast agent: safety, pharmacokinetics, and MR imaging. Radiology 1995; 195:785-792

10. Reimer P, Rummeny EJ, Shamsi K, Balzer T, Daldrup HE, Tombach B, et al. Phase II clinical evaluation of Gd-EOBDTPA: dose, safety aspects, and pulse sequence. Radiology 1996;199:177-183

11. Kim SH, Kim SH, Lee J, Kim MJ, Jeon YH, Park Y, et al. Gadoxetic acid-enhanced MRI versus triple-phase MDCT for the preoperative detection of hepatocellular carcinoma. AJR Am J Roentgenol 2009;192:1675-1681

12. Kim YK, Lee YH, Kwak HS, Kim CS, Han YM. Detection of liver metastases: Gadoxetic acid-enhanced three-dimensional MR imaging versus ferucarbotran-enhanced MR imaging. Eur J Radiol 2010;73:131-136

13. Lee MH, Kim SH, Kim H, Lee MW, Lee WJ. Differentiating focal eosinophilic infiltration from metastasis in the liver with gadoxetic acid-enhanced magnetic resonance imaging. Korean J Radiol 2011;12:439-449

14. Park Y, Kim SH, Kim SH, Jeon YH, Lee J, Kim MJ, et al. Gadoxetic acid (Gd-EOB-DTPA)-enhanced MRI versus gadobenate dimeglumine (Gd-BOPTA)-enhanced MRI for preoperatively detecting hepatocellular carcinoma: an initial experience. Korean J Radiol 2010;11:433-440

15. Ward J. New MR techniques for the detection of liver metastases. Cancer Imaging 2006;6:33-42

16. Ichikawa T, Saito K, Yoshioka N, Tanimoto A, Gokan T, Takehara $Y$, et al. Detection and characterization of focal liver lesions: a Japanese phase III, multicenter comparison between gadoxetic acid disodium-enhanced magnetic resonance imaging and contrast-enhanced computed tomography predominantly in patients with hepatocellular carcinoma and chronic liver disease. Invest Radiol 2010;45:133-141

17. Doo KW, Lee CH, Choi JW, Lee J, Kim KA, Park CM. “Pseudo washout" sign in high-flow hepatic hemangioma on gadoxetic acid contrast-enhanced MRI mimicking hypervascular tumor. AJR Am J Roentgenol 2009;193:W490-W496

18. Löwenthal D, Zeile M, Lim WY, Wybranski C, Fischbach F, Wieners $G$, et al. Detection and characterisation of focal liver lesions in colorectal carcinoma patients: comparison of diffusion-weighted and Gd-EOB-DTPA enhanced MR imaging. Eur Radiol 2011;21:832-840

19. Shimada K, Isoda H, Hirokawa Y, Arizono S, Shibata T, Togashi K. Comparison of gadolinium-EOB-DTPA-enhanced and 
diffusion-weighted liver MRI for detection of small hepatic metastases. Eur Radiol 2010;20:2690-2698

20. Motosugi U, Ichikawa T, Onohara K, Sou H, Sano K, Muhi A, et al. Distinguishing hepatic metastasis from hemangioma using gadoxetic acid-enhanced magnetic resonance imaging. Invest Radiol 2011;46:359-365

21. Ahn SJ, Kim MJ, Hong HS, Kim KA, Song HT. Distinguishing hemangiomas from malignant solid hepatic lesions: a comparison of heavily T2-weighted images obtained before and after administration of gadoxetic acid. J Magn Reson Imaging 2011;34:310-317

22. Ba-Ssalamah A, Uffmann M, Saini S, Bastati N, Herold C, Schima W. Clinical value of MRI liver-specific contrast agents: a tailored examination for a confident non-invasive diagnosis of focal liver lesions. Eur Radiol 2009;19:342-357

23. Danet IM, Semelka RC, Leonardou P, Braga L, Vaidean G, Woosley JT, et al. Spectrum of MRI appearances of untreated metastases of the liver. AJR Am J Roentgenol 2003;181:809817

24. Stern W, Schick F, Kopp AF, Reimer P, Shamsi K, Claussen CD, et al. Dynamic MR imaging of liver metastases with Gd-EOBDTPA. Acta Radiol 2000;41:255-262

25. Motosugi U, Ichikawa T, Morisaka H, Sou H, Muhi A, Kimura K, et al. Detection of pancreatic carcinoma and liver metastases with gadoxetic acid-enhanced MR imaging: comparison with contrast-enhanced multi-detector row CT. Radiology 2011;260:446-453

26. Choi JW, Lee JM, Kim SJ, Yoon JH, Baek JH, Han JK, et al. Hepatocellular carcinoma: imaging patterns on gadoxetic acid-enhanced MR Images and their value as an imaging biomarker. Radiology 2013;267:776-786

27. Sun HY, Lee JM, Shin CI, Lee DH, Moon SK, Kim KW, et al. Gadoxetic acid-enhanced magnetic resonance imaging for differentiating small hepatocellular carcinomas $(<$ or $=2 \mathrm{~cm}$ in diameter) from arterial enhancing pseudolesions: special emphasis on hepatobiliary phase imaging. Invest Radiol 2010;45:96-103

28. Chakraborty DP. Analysis of location specific observer performance data: validated extensions of the jackknife freeresponse (JAFROC) method. Acad Radiol 2006;13:1187-1193

29. Chakraborty DP, Berbaum KS. Observer studies involving detection and localization: modeling, analysis, and validation. Med Phys 2004;31:2313-2330

30. Vikgren J, Zachrisson S, Svalkvist A, Johnsson AA, Boijsen $M$, Flinck $A$, et al. Comparison of chest tomosynthesis and chest radiography for detection of pulmonary nodules: human observer study of clinical cases. Radiology 2008;249:10341041

31. Zheng B, Chakraborty DP, Rockette HE, Maitz GS, Gur D. A comparison of two data analyses from two observer performance studies using Jackknife ROC and JAFROC. Med
Phys 2005;32:1031-1034

32. Niekel MC, Bipat S, Stoker J. Diagnostic imaging of colorectal liver metastases with CT, MR imaging, FDG PET, and/or FDG PET/CT: a meta-analysis of prospective studies including patients who have not previously undergone treatment. Radiology 2010;257:674-684

33. Kulemann V, Schima W, Tamandl D, Kaczirek K, Gruenberger T, Wrba F, et al. Preoperative detection of colorectal liver metastases in fatty liver: MDCT or MRI? Eur J Radiol 2011;79:e1-e6

34. Park HS, Lee JM, Choi HK, Hong SH, Han JK, Choi BI. Preoperative evaluation of pancreatic cancer: comparison of gadolinium-enhanced dynamic MRI with MR cholangiopancreatography versus MDCT. J Magn Reson Imaging 2009;30:586-595

35. Lee JM, Zech CJ, Bolondi L, Jonas E, Kim MJ, Matsui 0, et al. Consensus report of the 4th International Forum for Gadolinium-Ethoxybenzyl-Diethylenetriamine Pentaacetic Acid Magnetic Resonance Imaging. Korean J Radiol 2011;12:403415

36. Kim YK, Ko SW, Hwang SB, Kim CS, Yu HC. Detection and characterization of liver metastases: 16 -slice multidetector computed tomography versus superparamagnetic iron oxide-enhanced magnetic resonance imaging. Eur Radiol 2006;16:1337-1345

37. Motosugi U, Ichikawa T, Nakajima H, Sou H, Sano M, Sano $\mathrm{K}$, et al. Imaging of small hepatic metastases of colorectal carcinoma: how to use superparamagnetic iron oxideenhanced magnetic resonance imaging in the multidetectorrow computed tomography age? J Comput Assist Tomogr 2009;33:266-272

38. Muhi A, Ichikawa T, Motosugi U, Sou H, Nakajima H, Sano K, et al. Diagnosis of colorectal hepatic metastases: Contrastenhanced ultrasonography versus contrast-enhanced computed tomography versus superparamagnetic iron oxideenhanced magnetic resonance imaging with diffusionweighted imaging. J Magn Reson Imaging 2010;32:1132-1140

39. Onishi H, Murakami T, Kim T, Hori M, Iannaccone R, Kuwabara $M$, et al. Hepatic metastases: detection with multi-detector row CT, SPIO-enhanced MR imaging, and both techniques combined. Radiology 2006;239:131-138

40. Schwartz L, Brody L, Brown K, Covey A, Tuorto S, Mazumdar $M$, et al. Prospective, blinded comparison of helical CT and CT arterial portography in the assessment of hepatic metastasis from colorectal carcinoma. World J Surg 2006;30:1892-1899; discussion 1900-1901

41. Holzapfel K, Eiber MJ, Fingerle AA, Bruegel M, Rummeny EJ, Gaa J. Detection, classification, and characterization of focal liver lesions: Value of diffusion-weighted MR imaging, gadoxetic acid-enhanced MR imaging and the combination of both methods. Abdom Imaging 2012;37:74-82 